\title{
REMARKS ON THE CLASSIFICATION OF RIEMANN SURFACES ${ }^{1}$
}

\author{
K. V. RAJESWARA RAO
}

1. Introduction. In [5] Royden constructed two Riemann surfaces to show that neither of the classes $O_{A B}$ and $O_{A D}$ of Riemann surfaces is quasiconformally invariant. In the present note it is shown how a slight modification of Royden's argument serves to establish the more general

TheOREM 1. No class of Riemann surfaces that contains $O_{L}$ and is contained in $O_{A D}$ is quasiconformally invariant.

This result is then used to prove

THEOREM 2. There is no inclusion relation between either of $O_{L}$ and $O_{L^{\prime}}$ and any one of $O_{H D}, O_{F D}$ and $O_{F B}$.

2. Notation and background. For any class $T$ of functions that can be considered on a Riemann surface, let $O_{T}$ stand for the class of all Riemann surfaces that do not admit nonconstant members of $T$. Denote by $L$ the class of Lindelöfian meromorphic functions (see [2]), i.e. meromorphic functions of bounded characteristic, and by $L^{\prime}$ the class of those members of $L$ which are pole-free. $A B$ and $A D$ denoting, respectively, the classes of bounded and Dirichlet-bounded analytic functions, it is known (see [2, p. 442] and [1, p. 201 and p. 256]) that

$$
O_{L} \subsetneq O_{L^{\prime}} \subsetneq O_{A B} \subsetneq O_{A D} .
$$

Let, now, $H D$ denote the class of Dirichlet-bounded harmonic functions and $F B$ and $F D$ signify, respectively, the classes of bounded and Dirichlet-bounded harmonic functions whose conjugate periods vanish along dividing cycles. It is clear that

$$
O_{H D} \subset O_{F D} \subset O_{A D}
$$

and it is known [7, p. 469] that

$$
O_{F B} \subset O_{F D} \text {. }
$$

3. The examples. Denote by $S$ the region of the complex plane obtained by punching out $z=0$ and $z=2$ from the disc $|z|<3$. Let

Received by the editors January 22, 1963 and, in revised form, March 25, 1963.

${ }^{1}$ Supported by U. S. Army Research Office (Durham) Grant No. DA-ARO(D)31-124-G40, University of California, Los Angeles. 
$W_{1}$ be a two-sheeted conformal cover of $S$ having branch points over $z=1 / n$ and $z=2-1 / n, n=2,3, \cdots, \infty$, and such that two curves of $W_{1}$ lie over the unit circle in $S$. Evidently, the projection map from $W_{1}$ to $S$ is an $A D$ function, i.e.

$$
W_{1} \notin O_{A D}
$$

Choose, now, $s$, an irrational multiple of $2 \pi$. Cut the upper sheet of $W_{1}$ over the unit circle $|z|=1$ and identify the point over $e^{i t}$ on the inside of the cut with the point over $e^{i t+i s}$ on the outside. Denote the resulting Riemann surface by $W_{2}$. We claim that

$$
W_{2} \in O_{L} \text {. }
$$

For, otherwise, let $f \in L$ on $W_{2}$. The projection map from the subregion $W_{2}^{\prime}$ of $W_{2}$ over the annulus $0<|z|<1$ into this annulus is of constant valence two. Hence, by a theorem of Heins [2, p. 444], $f$ is the quotient of two bounded analytic functions, say, $g$ and $h$, on $W_{2}^{\prime}$. But then, the argument of Royden $[5$, p. 6] shows that $g$ and $h$ are single valued functions of $z$ for $|z|<1$, and hence, so is $f$. Similarly, $f$ is a single valued function of $z$ for $1<|z|<3$. Hence, over $|z|=1$, we have $f\left(e^{i t}\right)=f\left(e^{i t+i s}\right)$.

Iterating we obtain that

$$
f\left(e^{i t}\right)=f\left(e^{i t+i m s}\right), \quad m=1,2, \cdots, \infty .
$$

Since the set of points $\left\{e^{i t+i m s}\right\}$ is dense in the unit circle, this implies that $f$ is a constant, which is a contradiction that establishes (5).

Now, the map $\phi: W_{1} \rightarrow W_{2}$, obtained by taking $\phi$ to be the identity except on the annulus over $1 / 2<|z|<1$ in the upper sheet of $W_{1}$ and $\phi\left(r e^{i t}\right)=r e^{i t+i s(2 r-1)}$ on this annulus, is a quasiconformal homeomorphism of $W_{1}$ onto $W_{2}$. This observation, together with (4) and (5), establishes Theorem 1.

Remarks. (i) As noted by Royden [5, p. 6], the map $\phi$ is "ultimately" conformal. Hence, the property of belonging to any of the classes of Theorem 1 is not a property of the ideal boundary [6, p. 58].

(ii) Examples of classes of Riemann surfaces for which Theorem 1 is applicable are $O_{L}, O_{L^{\prime}}, O_{A B}, O_{A D}$ and the classes $O_{A M_{\alpha}}[3$, p. 179].

(iii) $^{2}$ A construction, similar to the one above, carried out over the Riemann sphere instead of the disc $|z|<3$, yields an example to show that the class of parabolic Riemann surfaces admitting meromorphic functions of bounded valence is not preserved under quasiconformal maps.

\footnotetext{
${ }^{2}$ The author is indebted to the referee for pointing this out.
} 
4. Proof of Theorem 2. It is known $[6, \mathrm{p} .57]$ that $O_{F D}$ is quasiconformally invariant. This, in view of (2) and Theorem 1, implies that

$$
O_{L} \nsubseteq O_{F D}, \quad O_{L} \nsubseteq O_{H D} .
$$

Also, there exists [2, p. 441] a planar surface which does not belong to $O_{L}$, but belongs to $O_{A B}$. Since every cycle on a planar surface is dividing, this implies that

$$
O_{F B} \nsubseteq O_{L^{\prime}}
$$

Finally, in view of Theorem $26 \mathrm{H}$ of $[1$, p. 264] and (1), we have

$$
O_{H D} \nsubseteq O_{L^{\prime}} \text {. }
$$

Combining the first part of (1) with (6)-(8), we obtain the result. [4].

Note. An alternative proof of the second part of (6) was given in

\section{REFERENCES}

1. L. Ahlfors and L. Sario, Riemann surfaces, Princeton Univ. Press, Princeton N. J., 1960.

2. M. Heins, Lindelöfian maps, Ann. of Math. (2) 62 (1955), 418-446.

3. M. Parreau, Sur les moyennes des fonctions harmoniques et analytiques et la classification des surfaces de Riemann, Ann. Inst. Fourier (Grenoble) 3 (1951/1952), 103-197.

4. K. V. R. Rao, Lindelöfian meromorphic functions, Proc. Amer. Math. Soc. 15 (1964), 109-113.

5. H. L. Royden, Open Riemann surfaces, Ann. Acad. Sci. Fenn. Ser. A I. No. 249 (1958).

6. - On a class of null-bounded Riemann surfaces, Comment. Math. Helv. 34 (1960), 52-66.

7. L. Sario, An extremal method on arbitrary Riemann surfaces, Trans. Amer. Math. Soc. 73 (1952), 459-470.

University of California, Los Angeles 